\title{
MORE ON THE AXIAL ANOMALY IN SUPERSYMMETRIC YANG-MILLS THEORY
}

\author{
D.R.T. JONES \\ Department of Physics, University of Michigan, Ann Arbor, MI 48109, USA
}

Received 3 January 1983

\begin{abstract}
The compatibility of the Adler-Bardeen theorem with the supermultiplet containing the axial, trace, and supercurrent anomalies in $N=1$ supersymmetric Yang-Mills theory is re-examined (in component form). It is argued that consideration of the off-shell form of the supermultiplet removes the apparent paradox, at least at the two-loop level.
\end{abstract}

In this short note the question of the compatibility of the Adler-Bardeen (AB) theorem [1] and supersymmetry in supersymmetric Yang-Mills theory (SSYM) is re-examined. As is well known [2-5] in SSYM the axial, the trace of the energy-momentum tensor and the supersymmetry $(\gamma \cdot S)$ anomalies form the $G, F, \psi$ components respectively of a WessZumino multiplet $(A B \psi F G)$. The possible conflict between the $\mathrm{AB}$ theorem and the supermultiplet nature of the anomalies was discussed by Grisaru [6]. A recent calculation [7] verified that, providing the Ward identities are consistently imposed, the $\mathbf{A B}$ theorem is valid (at the two-loop level) in both abelian and non-abelian gauge theories using either conventional or supersymmetric dimensional regularization (CDR or SDR). The significance of this result for the SSYM case was unclear, however, since use of dimensional regularization necessitates use of a nonanti-commuting $\gamma^{5}$ which leads to modifications of the supersymmetry Ward identities. Here we make a simple observation which shows that the $A B$ theorem does not conflict with the supermultiplet structure of the anomalies (at least at the two-loop level).

The lagrangian for SSYM (in the Wess-Zumino gauge) forms the $F$ component of a Wess-Zumino chiral supermultiplet:

$A=\frac{1}{4} \bar{\lambda} \lambda, \quad B=\frac{1}{4} \bar{\lambda} \mathrm{i} \gamma^{5} \lambda$ $\psi=\frac{1}{4} \sigma^{\mu \nu} \gamma^{5} F_{\mu \nu} \lambda+\frac{1}{2} D \lambda$, $F=-\frac{1}{4} F_{\mu \nu}^{2}+\frac{1}{2} \mathrm{i} \tilde{\lambda} \gamma_{\mu} D^{\mu} \lambda+\frac{1}{2} D^{2}$, $G=-\frac{1}{8} \epsilon_{\mu \nu \alpha \beta} F^{\mu \nu} F^{\alpha \beta}+\frac{1}{4} \partial_{\mu}\left(\bar{\lambda} \gamma_{\mu} \gamma^{5} \lambda\right)$.
(Our conventions are those of Bjorken and Drell.) Note that (1) is an off-shell multiplet: it is not necessary to use the equations of motion to verify the transformation properties.

In ref. [7] it was shown that, in accordance with the $\mathrm{AB}$ theorem,

$\partial_{\mu} J_{\mu}^{5}=\partial_{\mu}\left[\frac{1}{2} \bar{\lambda} \gamma_{\mu} \gamma^{5} \lambda\right]=+\frac{1}{2}\left[C_{2}(G) g^{2} / 16 \pi^{2}\right] F \widetilde{F}$.

From (1) and (2) we find

$$
\begin{aligned}
& \partial_{\mu} J_{\mu}^{5}=\left[-3 C_{2}(G) / 16 \pi^{2}\right] g^{2} \\
& \quad \times\left\{1-2\left[C_{2}(G) / 16 \pi^{2}\right] g^{2}\right\}^{-1} \frac{4}{3} G,
\end{aligned}
$$

and we see that, since

$$
\begin{aligned}
& \beta(g)=\left[-3 C_{2}(G) / 16 \pi^{2}\right] g^{3}-\left[6 C_{2}(G)^{2} /\left(16 \pi^{2}\right)^{2}\right] g^{5} \\
& \quad+\mathrm{O}\left(g^{7}\right),
\end{aligned}
$$

we have

$\partial_{\mu} J_{\mu}^{5}=[\beta(g) / g] \frac{4}{3} G$

(to two-loop order at least).

Thus up to the two-loop level the association of the axial current $J_{\mu}^{5}$ with $\theta_{\mu}^{\nu}$ in a supermultiplet is not problematic. What happens at higher orders remains an open question.

According to ref. $[8], \beta(g)$ is given by

$$
\begin{aligned}
& \beta(g)=-3\left[C_{2}(G) / 16 \pi^{2}\right] g^{3}-6\left[C_{2}(G) / 16 \pi^{2}\right]^{2} g^{5} \\
& -21\left[C_{2}(G) / 16 \pi^{2}\right]^{3} g^{7},
\end{aligned}
$$


when calculated using SDR. Thus assuming this calculation is correct the simple form for $\beta(g)$ suggested by (3) is invalid at the three-loop level. It should be noted, however, that SDR has problems [9] (in the component form at least), and also it is well known that at the three-loop level $\beta(g)$ depends on the subtraction scheme. Thus it is not obvious that there is really a problem at higher orders. Of course we have been cavalier with regard to the question of renormalization of the various quantities in the above equations. We do not believe, however, that a careful treatment would change the main conclusion, which is that including the terms which vanish by the equations of motion in (1) leads to a resolution of the anomaly paradox at the two-loop level.

I wish to thank S. Adler, G. Bonneau and K. Sibold for stimulating discussions and correspondence. This work was supported in part by the Department of Energy.

\section{References}

[1] S.L. Adler and W.A. Bardeen, Phys. Rev. 182 (1969) 1517; S.L. Adler, R.W. Brown, T.F. Wong and B.-L. Young, Phys. Rev. D4 (1971) 1787;

S.L. Adler, in: Lectures on elementary particles and quantum field theory (Brandeis, 1970) eds. S. Deser, M. Grisaru and $\mathrm{H}$. Pendleton.

[2] S. Ferrara and B. Zumino, Nucl. Phys. B87 (1975) 207.

[3] T. Curtright, Phys. Lett. 71B (1977) 185.

[4] L.F. Abbott, M.T. Grisaru and H.J. Schnitzer, Phys. Rev. D16 (1977) 2995; Phys. Lett. 71 B (1977) 161; T. Hagiwara, S.Y. Pi and H.S. Tsao, preprint COO-22328267 (1978), unpublished.

[5] O. Piguet and K. Sibold, Nucl. Phys. B 196 (1982) 428, 447.

[6] M. Grisaru, in: Recent developments in gravitation (Cargese, 1980) eds. M. Levy and S. Deser (Plenum, New York).

[7] D.R.T. Jones and J.P. Leveille, Phys. Lett. 109B (1982) 449; Nucl. Phys. B206 (1982) 473

[8] L.N. Avdeev, G.A. Chochia and A.A. Vladimirov, Phys. Lett. 105B (1981) 272.

[9] L.V. Avdeev and O.V. Tarasov, Phys. Lett. 112B (1982) 356. 